\title{
Orbital Lymphoma about 4 Cases
}

Coulibaly $\mathrm{K}^{1}$, Traoré $\mathrm{S}^{2}$, Samaké $\mathrm{D}^{3^{*}}$, Traoré $\mathrm{H}^{2}$, Bathily $\mathrm{M}^{4}$, Kané $\mathrm{AST}^{5}$, Oukerroum $\mathrm{A}^{1}$, Slimani $\mathrm{F}^{1}$

\author{
${ }^{1}$ Department of Stomatology and Maxillofacial Surgery, Hôpital du 20 Août Casablanca - Marocco \\ ${ }^{2}$ Department of Stomatology and Maxillofacial Surgery, CHU-CNOS-Bamako, Mali \\ ${ }^{3}$ ENT and Head and Neck Surgery Department, Commune V Reference Health Center, Bamako, Mali \\ ${ }^{4}$ Haemato-Oncology Department, CHU Point-G- Bamako, Mali \\ ${ }^{5}$ Department of Odontology, Bamako Military Hospital, Mali
}

DOI: $10.36347 /$ simcr.2020.v08i04.001

| Received: 23.03.2020 | Accepted: 31.03.2020 | Published: 05.04.2020

*Corresponding author: Dr Samaké Djibril

Abstract

We report four cases of exophthalmos related to malignant lymphoma, highlighting the value of surgical biopsy. These were four patients aged 33, 50, 55 and 62 respectively. Unilateral exophthalmos was the reason for consultation in all cases. The diagnosis of non-Hodgkin's lymphoma was suspected by imaging and confirmed by pathological examination of surgical biopsies. It was type B lymphoma in 3 cases and TNK type lymphoma in 1 case. They all benefited from polychemotherapy. The evolution was marked by the regression of the tumor and exophthalmos. These cases show that the clinical polymorphism of malignant lymphomas is extreme. They can cause diagnostic problems and delay treatment. Hence the interest of the histological examination of a tissue biopsy.

Keywords: Lymphomas; Orbit; Biopsy.

Copyright @ 2020: This is an open-access article distributed under the terms of the Creative Commons Attribution license which permits unrestricted use, distribution, and reproduction in any medium for non-commercial use (NonCommercial, or CC-BY-NC) provided the original author and source are credited.

\section{INTRODUCTION}

Lymphomas are malignant monoclonal proliferations of $\mathrm{B}$ or $\mathrm{T}$ lymphoid cells at different stages of their maturation and less often natural Killer $\mathrm{T}$ cells (TNK). They can be divided into 2 main groups: Hodgkin lymphoma and non-Hodgkin lymphoma (NHL) [1]. Orbital lymphomas account for approximately 1 to $2 \%$ of non-Hodgkin's lymphomas $[2,3]$. And they constitute between 6 and $8 \%$ of orbital tumors and 5 to $15 \%$ of extra-lymph node lymphomas [2-4]. They can come from the eyelid, the lacrimal glands or the conjunctiva [4]. Clinical and radiological signs are useful for diagnostic suspicion. Because they are not specific, they can easily be mistaken for chronic inflammatory disease or other tumors of the orbit or appendages [4, 5, 6]. Another differential diagnosis is thyroid orbitopathy [4]. Hence the need for a histological examination [5]. The majority are MALT type B marginal cell lymphomas (70-80\%) [6-8]. The other subtypes are follicular lymphomas (5-10\%), diffuse large B cell and mantle cell lymphomas (9\%) [8]. Treatment depends on the histological grade, the size of the tumor and the possible systemic extension [9]. It is mainly based on chemotherapy and more or less irradiation therapy $[7,10]$. Different techniques are described for having a histological diagnosis $[5,6]$. The choice could be difficult [6]. The aim of this work is to report the medical observations of four patients with unilateral exophthalmos related to malignant lymphoma and to highlight the value of surgical biopsy

\section{Observations}

We report the medical observations of four patients aged 33, 50, 55 and 62 years respectively. They presented with orbital lymphoma; diagnosed in the department of stomatology and maxillofacial surgery at the August 20 hospital in Casablanca. There was no specific medical history.

The sign of appeal was progressive exophthalmos, which was left paraxial in three patients (Figure-1) and right paraxial in one patient with chronic dacryocystitis.

The decrease in visual acuity was noted in 1 case. Two patients had an eye movement disorder with upper vertical diplopia associated with eye pain.

The left orbital ultrasound performed in three patients showed the presence of a global swelling of the periocular envelope of heterogeneous hypoechogenic aspect with infiltration and thickening of the neighboring structures. 
Computed tomography (Figure-2) was performed on all patients. It found a localization on the outer wall of the orbit in 3 patients and on the inner wall in one patient. The mass sitting at the level of the external wall of the orbit was of tissue density moderately taking the contrast agent with grade I and II exophthalmos. The one sitting at the level of the internal wall showed a mass of tissue density taking up the contrast product.

Magnetic Resonance Imaging (MRI), performed in a patient, showed the presence of a large expansive left orbital expansive process of extraconal seat centered by the internal right muscle; it was poorly limited, in $\mathrm{T} 1$ hypo signal and $\mathrm{T} 2$ hyper signal with intense and heterogeneous contrast enhancement delimiting a central area of necrosis. This process pushed the eyeball back and forth, with an invasion of the inner wall.

A surgical biopsy by approaching the external wall of the orbit with deposit of it was performed in three patients (Figure 3 and 4). It allowed good exposure of the tumor mass, which was largely resected (Figures 5 and 6).

The extended lateral-nasal approach to the root of the eyebrow was performed in one patient.

The anatomo-pathological and immunohistochemical study carried out concluded that diffuse non-Hodgkin B-cell large lymphoma in 3 patients and TNK cell lymphoma in one patient.

Thoracoabdominal CT scan was performed in all patients. She had not revealed secondary locations.

They were all sent to the Hematology department where bone marrow biopsies performed returned to normal.

The three patients with B lymphoma, benefited from multi-chemotherapy cures by the R-CHOP protocol. The patient with TNK cell lymphoma benefited from SMILE multidrug cures.

The polychemotherapy cure instituted lasted 6 months. The evolution was marked by the disappearance of the tumor mass and symptoms in all the patients. After one year of post-operative follow-up there are no signs of recurrence.

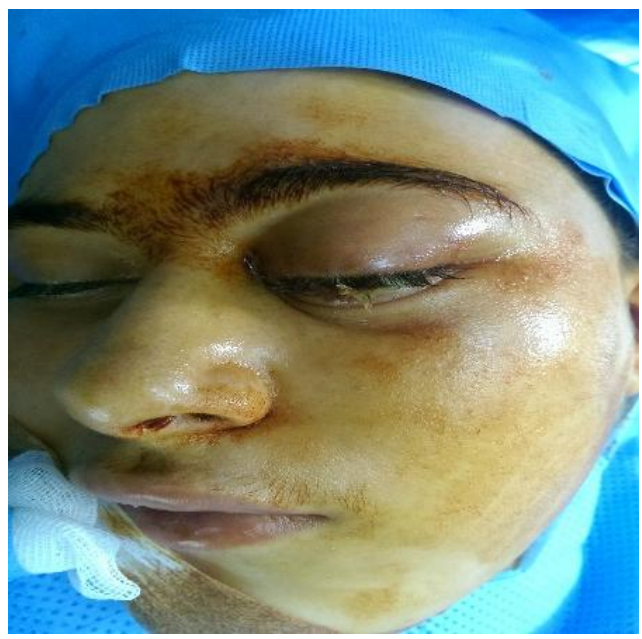

Fig-1: Patient with left orbital mass

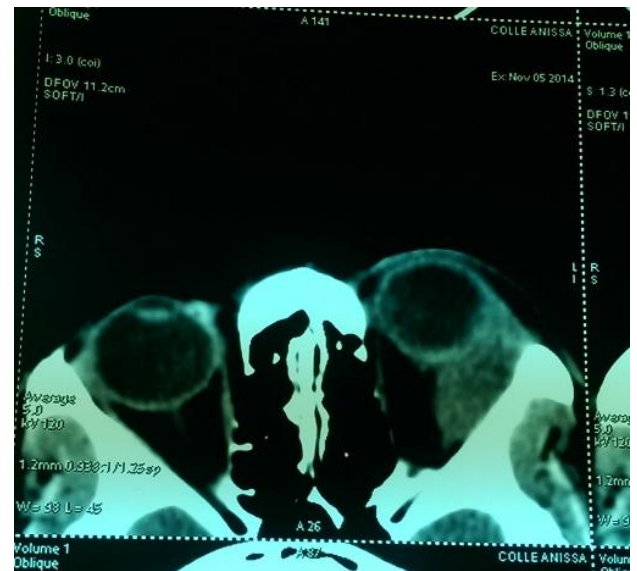

Fig-2: CT of the face in axial section showing a left exophthalmos and a tissue mass at the outer wall of the orbit
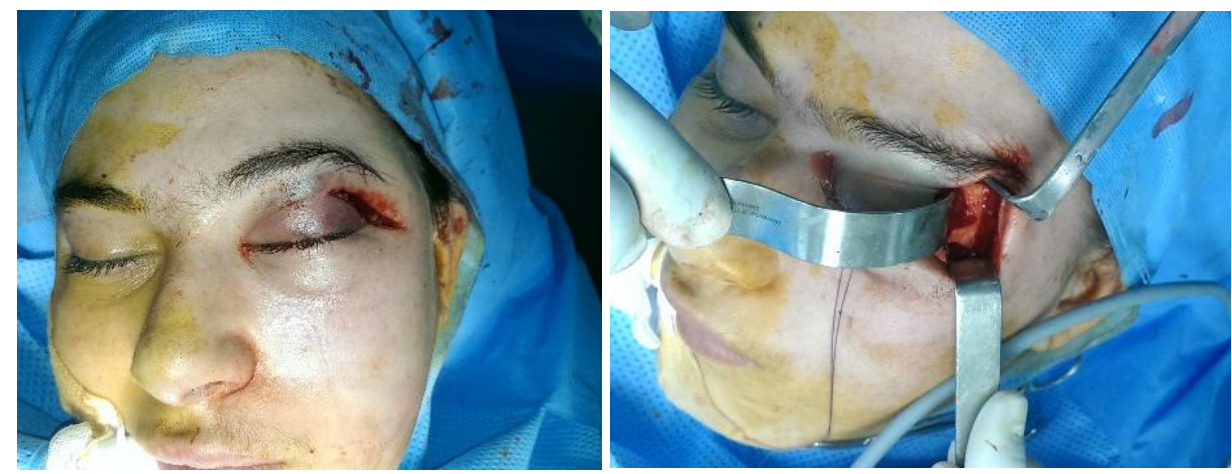

Fig 3 and 4: Approach of the external wall of the orbit with deposit of it 


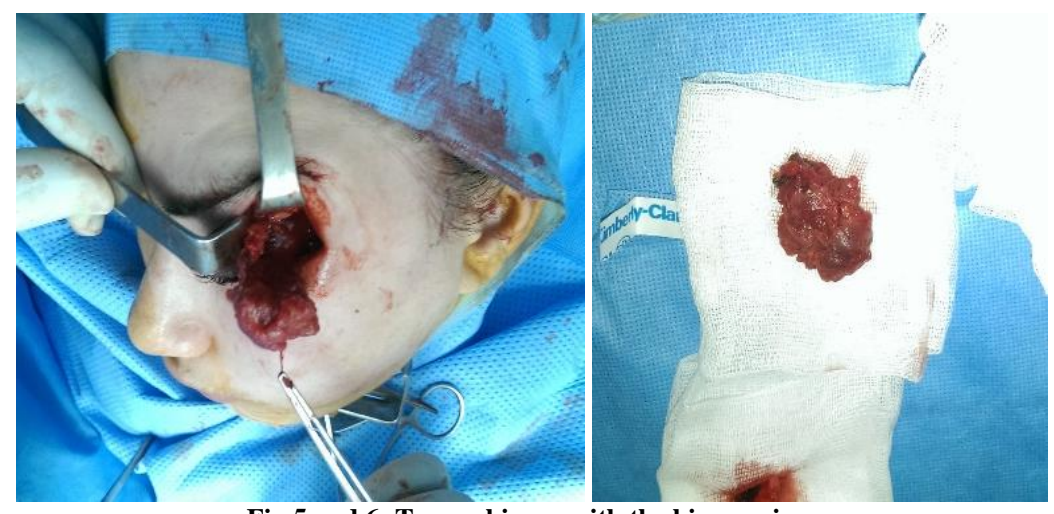

Fig 5 and 6: Tumor biopsy with the biopsy piece

\section{DISCUSSION}

Lymphomas represent $11 \%$ of all orbit tumors and $55 \%$ of its malignant tumors [6, 7]. They are generally present in the 50-70 age group, with a slight preponderance of women [7, 8]. In our study, these were women $33,50,55$ and 62 without a history of lymphoma. In the literature, most orbital lymphomas are in the form of a painless, slow growing orbital mass with increasing exophthalmos. Diplopia and reduced eye mobility are also common $[3,7,8]$. We found the same signs. Vision is rarely affected [8]. The signs are often one-sided. However, bilateralism is observed in $5 \%$ of cases [7]. In $25 \%$ of cases, where the conjunctiva is involved, patients present with salmon-red patches or swollen conjunctiva [4]. Due to its relatively benign course and the non-specificity of the signs, the diagnosis and therefore the treatment of orbital lymphomas are often delayed [8]. It can also be asymptomatic by chance discovery during a CT scan [3]. In imaging the lesions most often sit in the superlateral framing of the orbit $[2,6,7]$. They are generally extra-conical and are almost always unilateral $[2,6]$. They infiltrate the upper right muscle, the lateral right muscle, the lacrimal gland and the eyelid. They rarely reach the lower quadrants and the optic nerve [2]. Orbital lymphoma is characterized by a molding around the globe, reflecting irregular infiltration of the orbital structures, and only seems to be defined in $20 \%$ of cases [6]. MRI is the examination of choice which provides excellent details on the adjacent orbital structures and the state of the optical apparatus [8]. The images are iso-intense compared to the extraocular muscles on the images balanced in T1 and T2. In our case we made the same observations. These images and this clinical presentation have several similarities with other totally different lesions [6]. In some situations, patients may have a history of lymphoma or develop systemic lymphoma during the course of evolution [11]. The extension balance sheet is essential. It must concern the superficial and deep lymph node areas (abdominothoracic scanner), bone marrow, and hepatic assessment [12]. In the present study, the location was orbital with no other secondary locations. To have a histological diagnosis, different techniques are used. And Choosing the appropriate surgical approach between fine needle aspiration biopsies, intraoperative biopsies and total tumor removal may be a challenge [5, 6]. The Gold-Standard is the fine needle aspiration biopsy or the "Core needle biopsy (CNB) or Fine needle aspiration biopsy (FNAB)" fine needle biopsy under ultrasound or computed tomography guidance. However, it is often limited in terms of challenge for lesions located in the posterior part of the orbit [13]. These minimally invasive techniques require an experienced practitioner, well trained at the risk of damage to the globe, the optic nerve and false results. But also if it is not done in depth the result will only be an inflammatory infiltrate surrounding the tumor itself [14]. Histological and cytological reading also recommends a well-trained and accustomed pathologist [15]. In our study we recommended open surgical biopsy. This allowed good macroscopic visibility of the tumor and resection with a sample of important tumor tissue for histological and immunohistochemical studies. Open surgical biopsy is recommended by most authors. It is usually made through a small incision under local anesthesia. Deep orbital lesions are less common. These cases generally require general anesthesia [5]. The assessment of extension makes it possible to classify the lymphoma according to the classification of Ann Arbor which will guide the treatment and predict the prognosis [8, 9]. This treatment depends on the degree of malignancy, its aggressiveness and the existence or not of a systemic lymphoma [9]. The majority of orbital lymphomas are low grade $(84 \%)$, and a small proportion $(16 \%)$ are high grade (aggressive) [4]. Other classification systems such as that of the eighth edition of the AJCC exist and are more suitable [3]. Treatment options include surgery, irradiation therapy and chemotherapy. The role of surgery is limited to biopsy in the majority of cases.

The surgical approach depends on the location of the lesion: lateral orbitotomy for laterally located tumors, trans-conjunctival approach for medial, basal and extra-intraconal lesions and intra-extradural pterian procedures for lesions at the apex of the 2 orbits or those with intracranial extensions [8]. Primary irradiation therapy (usually 25-35 Gy) is the standard treatment for stage IE (low-grade isolated orbital lymphoma) [8, 10]. In case of isolated high-grade orbital lymphoma: 3 cycles of $\mathrm{CHOP}$ induction 
chemotherapy followed by irradiation therapy [10]. In our study all of our patients had an isolated orbital location. They benefited exclusively from multidrug therapy without irradiation therapy. The evolution at one year was marked by the regression of the masses and the disappearance of the symptoms. The patient with TNK-type lymphoma is still under regular surveillance.

\section{Conclusion}

Non-Hodgkin's orbital malignant lymphomas represent a fairly large proportion of orbital tumors. Their diagnoses are often delayed because they are confused with chronic orbital inflammation and other tumors. The present study indicates that the signs are polymorphic. And that surgical biopsy for appropriate treatment is essential immediately in these cases. Statement for ethics: written informed consent was obtained from the patient for the purpose of publication.

Conflicts of Interest: The authors declare that they have no conflicts of interest.

\section{REFERENCES}

1. Kirkegaard MM, Coupland SE, Prause JU, Heegaard S. Malignant lymphoma of the conjunctiva. Survey of ophthalmology, 2015; 60:444-458.

2. Gema P, Carles M, Fina C, Amadeo M. Insights Imaging, 2012;3:337-344.

3. Maheshwari A, Finger PT. Cancers of the eye. Cancer and Metastasis Reviews. Springer Nature, 2018;18.

4. Törők-Vistai T, Bojan A, Cucuianu A, Zsoldos A. Primary non-hodgkin lymphoma of the orbit presenting with massive bilateral periorbital tumors. Clujul Medical, 2013;86(4): 380-382.

5. Briscoe D, Safieh C, Ton Y, Shapiro H, Ehud IA, Kidron D, Ehud IA, Kidron D. Characteristics of orbital lymphoma: a clinicopathological study of 26 cases. Int Ophthalmol, 2018;38:271-277.

6. Diego S, Elefante A, Matarazzo F, Panico A,
Ferrara M, Tranfa F. Orbital lymphoma mimicking lacrimal gland pleomorphic adenoma. Case Rep Ophthalmol, 2013;4:109-113.

7. Ashwin UB, Kapil J, Vijendra KJ. A large primary orbital lymphoma with proptosis: A case report and review. Surg Neurol Int, 2018;9:249.

8. 8: Prasad GL, Hegde A, Menon G, Mathew M. Primary Orbital Follicular Lymphoma: A Case Report and Review. Journal of Clinical and Diagnostic Research. Mar 2017;11(3): XD01XD03.

9. Eckardt AM, Lemound J, Rana M., Gellrich NC. Orbital lymphoma: diagnostic approach and treatment outcome. World Journal of Surgical Oncology, 2013;11:73.

10. Sachsman S, Flampouri S, Li Z, Lynch J, Mendenhall NP, Hoppe BS. Proton therapy in the management of non-Hodgkin lymphoma. Leukemia \& Lymphoma, September 2015; 56(9):2608-2612.

11. Sagiv O, Thakar SD, Manning JT, Kandl TJ, Fayad LE, Nathan F, Hagemeister FB, Fanale MA, Pinnix CC, Samaniego F, Esmaeli B. Prevalence of a histologic change of ocular adnexal lymphoma in patients with a history of lymphoma. Ophthal Plast Reconstr Surg, 2018;20(20):1-4.

12. Bella-Hiag AL, Ebana C, Sede Mbakop. Le lymphome malin orbito-sinusien. A propos d'un cas. Médecine d'Afrique Noire, 2000;47(1):52-53.

13. Ren J, Yuan Y, Wu Y, Tao X. Differentiation of orbital lymphoma and idiopathic orbital inflammatory pseudotumor: combined diagnostic value of conventional MRI and histogram analysis of ADC maps. BMC Medical Imaging 2018;18:6.

14. Eenhorst CA, Laban KG, Leguit RJ, Radstake RD, Kalmann R. Orbital lymphomas missed by first biopsies of orbital masses. Acta Ophthalmologica, 2017:1-2.

15. Christopher E, Richard B, Wilder, Chul S, Mark A, Fernando F, James DC. Clinical stage IEA IIEA orbital lymphomas: outcome in the era of modern staging and treatment, Radiotherapy and Oncology, 2001;59(2):145- 151. 